Published in final edited form as:

Anal Chem. 2019 October 15; 91(20): 12917-12927. doi:10.1021/acs.analchem.9b02904.

\title{
Direct in Vivo Electrochemical Detection of Resting Dopamine Using Poly(3,4-ethylenedioxythiophene)/Carbon Nanotube Functionalized Microelectrodes
}

\author{
Ian Mitchell Taylor ${ }^{\dagger, \ddagger}$, Nikita Anurag Patel ${ }^{\dagger}$, Noah Chaim Freedman ${ }^{\dagger}$, Elisa Castagnola ${ }^{\dagger}$, \\ Xinyan Tracy Cui ${ }^{*} \dagger$ \\ †Department of Bioengineering, University of Pittsburgh, Pittsburgh, Pennsylvania 15261, United \\ States \\ ¥Department of Chemistry, Saint Vincent College, Latrobe, Pennsylvania 15650, United States
}

\section{Abstract}

Dopamine (DA) is a monoamine neurotransmitter responsible for the maintenance of a variety of vital life functions. In vivo DA signaling occurs over multiple time scales, from subsecond phasic release due to dopamine neuron firing to tonic release responsible for long-term DA concentration changes over minutes to hours. Due to the complex, multifaceted nature of DA signaling, analytical sensing technology must be capable of recording DA from multiple locations and over multiple time scales. Decades of research has focused on improving in vivo detection capabilities for subsecond phasic DA, but the accurate detection of absolute resting DA levels in real time has proven challenging. We have developed a poly(3,4-ethylenedioxythiophene) (PEDOT)-based nanocomposite coating that exhibits excellent DA sensing capabilities for resting DA. PEDOT/ functionalized carbon nanotube (PEDOT/CNT)-coated carbon fiber microelectrodes (CFEs) are capable of directly measuring resting DA using square wave voltammetry (SWV) with high sensitivity and selectivity. Incorporation of a PEDOT/CNT coating significantly increases the sensitivity for the detection of resting DA by a factor of 422 . SWV measurements performed at PEDOT/CNT-functionalized CFEs implanted in the rat dorsal striatum reveal the absolute basal DA concentration to be $82 \pm 6 \mathrm{nM}$. Systemic administration of the dopamine transporter inhibitor nomifensine increases resting DA to a maximum $207 \pm 16 \mathrm{nM}$ at $28 \pm 2$ min following injection. PEDOT/CNT was also functionalized onto individual gold electrode sites along silicon microelectrode arrays (MEAs) to produce a multisite DA sensing electrode. MEA implantation allows for the quantification of basal DA from different brain regions with excellent spatial

\footnotetext{
*Corresponding Author: xic11@ pitt.edu. Phone: 412-383-6672.

Author Contributions

I.M.T. was responsible for project conceptualization, design, data collection, data analysis, and manuscript preparation. N.A.P. and N.C.F. contributed equally to the project and were responsible for data collection, data analysis, and provided valuable mechanistic insight. E.C. was responsible for data collection and data analysis for contaminant experiments. X.T.C. supervised the project and edited the manuscript.

Supporting Information

The Supporting Information is available free of charge on the ACS Publications website at DOI: 10.1021/acs.analchem.9b02904. Detailed methodology for CFE preparation, CNT preparation, scanning electron microscopy, and square wave voltammetry, PEDOT/ CNT-functionalized CFEs postexplantation, PEDOT/CNT coating on MEA, and in vivo experimentation and data analysis (PDF) The authors declare no competing financial interest.
} 
resolution. SWV detection paired with PEDOT/CNT functionalization is highly adaptable and shows great promise for tonic DA detection with high spatial and temporal resolution.

\section{Graphical Abstract}
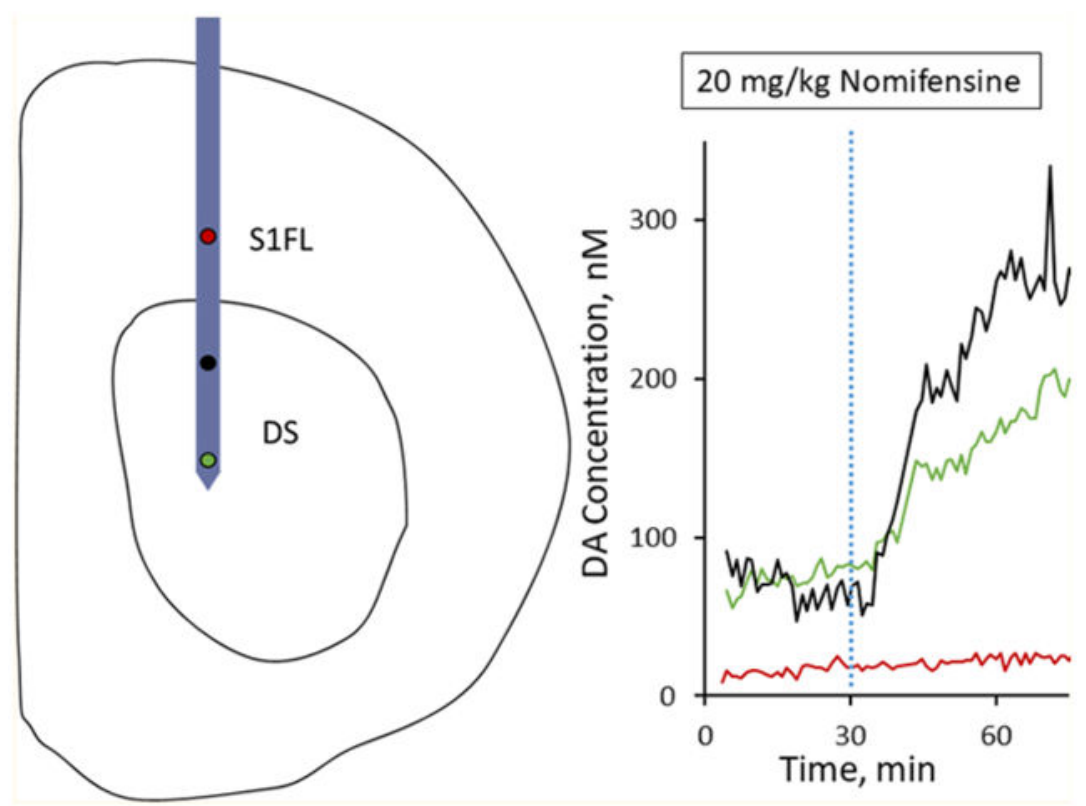

Tonic and phasic dopamine (DA) neurotransmission are responsible for the regulation of a myriad of vital life functions. ${ }^{1-6}$ Phasic DA release induces transient, subsecond fluctuations in extracellular DA, whereas tonic firing maintains basal DA levels that serve to regulate phasic firing. ${ }^{7,8}$ It is widely believed that preserving tonic and phasic firing is key to maintaining healthy neuronal functionality. Dysfunction in the regulation of tonic and phasic DA firing has been implicated in the onset of devastating neurological disorders, such as schizophrenia. ${ }^{6}$ As such, it is absolutely necessary to consider both tonic and phasic DA signaling events when studying DA signaling pathways.

DA is an electroactive compound capable of reversible oxidation to dopamine- $o$-quinone (DAoQ) upon application of a sufficient potential. Electrochemical measurement of the current associated with this reversible reaction permits real-time, direct measurement of DA concentration. Fast scan cyclic voltammetry (FSCV) performed at carbon fiber microelectrodes (CFEs) has been considered the gold standard for the in vivo measurement of phasic DA signaling events for decades. ${ }^{9}, 10$ As a differential detection method, FSCV requires background subtraction of large capacitive currents which are unstable over the minute time scale. This has prevented the use of FSCV for the detection of absolute resting DA concentrations. The only way to use FSCV for the quantification of basal DA is to induce pharmacological manipulation of the basal level. FSCV measurement during the direct infusion of kynurenate, a broad-spectrum antagonist of ionotropic glutamate receptors, suggests that the basal DA concentration in the dorsal striatum is above $2.6 \mu \mathrm{M} .{ }^{11}$ Microdialysis is widely used to observe resting DA in vivo. ${ }^{12-18}$ In fact, no-net-flux microdialysis has been used to estimate the basal DA concentration in vivo to be around 2.5- 
$15 \mathrm{nM} .{ }^{19-23}$ However, implantation of large microdialysis probes $(\sim 200 \mu \mathrm{m}$ o.d. $\times 1 \mathrm{~mm})$ results in substantial tissue damage that greatly diminishes extraction efficiency which produces an underestimation of in vivo quantification and prevents chronic measurement beyond 5-10 days. ${ }^{20,24-31}$ Several electrochemical approaches have been developed to measure drug-induced changes in tonic DA over several hours at CFEs. This was accomplished by utilizing charge balancing waveforms, ${ }^{32}$ controlled DA adsorption waveforms, ${ }^{33-36}$ convolution-based FSCV nonfaradaic current removal, ${ }^{37,38}$ multiple cyclic square wave voltammetry, ${ }^{39}$ and differential normal pulse voltammetry. ${ }^{40-42}$ Fast scan controlled adsorption voltammetry (FSCAV) and convolution-based FSCV were capable of estimating basal DA concentration in nucleus accumbens of both mice ( $90 \pm 9 \mathrm{nM}, \mathrm{FSCAV})$ and rats ( $41 \pm 13 \mathrm{nM}$, convolution-based FSCV), respectively. ${ }^{35,38}$ Multiple cyclic square wave voltammetry (m-CSWV) determined the tonic dopamine concentration to be $120 \pm 18$ $\mathrm{nM}$ in the rat striatum. ${ }^{39}$ Differential normal pulse voltammetry (DNPV) determined the basal DA concentration to be $26 \pm 8 \mathrm{nM}$ in the pargyline-pretreated rat striatum. ${ }^{40}$ These studies display the feasibility of using electrochemical methods to measure tonic DA.

Our laboratory has developed a conductive polymer coating consisting of functionalized carbon nanotube doped poly(3,4-ethylenedioxythiophene) (PEDOT/CNT). PEDOT/CNT can be controllably deposited onto both macro- -43 and microelectrodes ${ }^{44-46}$ via electropolymerization, resulting in increased effective surface area, increased charge storage capacity (increased capacitance), and decreased electrode impedance. ${ }^{43-46}$ PEDOT/CNTfunctionalized microelectrodes have exhibited excellent in vivo performance for both electrophysiological recording 44,45 and electrical stimulation ${ }^{46,47}$ over several months of chronic implantation. Furthermore, PEDOT/CNT-functionalized carbon paste macroelectrodes are highly sensitive and selective for the electrochemical detection of resting DA in vitro via differential pulse voltammetry. ${ }^{43}$ In this study we incorporate PEDOT/CNT coatings onto both implantable CFEs and gold electrode sites along singleshank silicon-based microelectrode arrays (MEAs) to produce a highly adaptable and robust new electrode technology. Pairing PEDOT/CNT-functionalized microelectrodes in an array with a highly optimized in vivo square wave voltammetry (SWV) protocol allows for the direct quantification of resting (tonic) DA in the rat with excellent spatial and temporal resolution. The power of this technology is clearly evident in the results of a proof-ofprinciple experiment detailing the first reported time-correlated, multisite quantification of basal DA in the brain by recording at multiple PEDOT/CNT-functionalized electrode sites along a single silicon MEA. Multisite detection of in vivo resting DA represents a substantial improvement in the current state of the art of neurochemical sensing and could serve to enhance our understanding of healthy and disease state neurological function.

\section{MATERIALS AND METHODS}

\section{Electrode Preparation.}

CFEs were produced using previously described methods. ${ }^{48-51}$ Details are provided in the Supporting Information. Silicon-based neural recording MEAs were obtained from Diagnostic Biochips (Glen Burnie, MD, U.S.A.). MEAs consisted of a single silicon shank $(90 \mu \mathrm{m} \times 15 \mu \mathrm{m} \times 7 \mathrm{~mm})$ with 16 individual $37 \mu \mathrm{m}$ diameter gold electrode sites centered 
and equally spaced along the shank at $200 \mu \mathrm{m}$ increments ( $3 \mathrm{~mm}$ total electrode spacing).

Please see our previous publication for more details regarding MEA fabrication. ${ }^{52}$

CNT functionalization was conducted using previously established methods. ${ }^{43-46,51}$ Details are provided in the Supporting Information. Polymerization solution was freshly prepared immediately prior to electrode polymerization by first suspending $1 \mathrm{mg} / \mathrm{mL}$ of CNTs in Nanopure water via bath sonication, and then adding $1 \mu \mathrm{L} / \mathrm{mL}$ of 3,4ethylenedioxythiophene (EDOT, Sigma Adrich, St. Louis, MO, U.S.A.). The solution was then vortexed prior to undergoing $45 \mathrm{~min}$ of pulsed probe sonication ( $2 \mathrm{~s}$ on, $1 \mathrm{~s}$ off, $20 \%$ power, $1.6 \mathrm{~mm}$ tip, Q500 Sonicator, QSonica LLC, Newtown, CT, U.S.A.). Probe sonication aids in the dissolution of EDOT into the solution and serves to further break up the CNTs into smaller units. ${ }^{51,53,54}$ Following probe sonication, the solution was again vigorously mixed immediately prior to electropolymerization. Identical electropolymerization procedures were used for both CFEs and $37 \mu \mathrm{m}$ diameter gold electrode sites located along single-shank, silicon MEAs. Electrodes were polymerized via chronocoulometry using a three-electrode design with either an individual CFE or an individual gold electrode located along the MEA as a working electrode, a platinum sheet counter electrode, and a $\mathrm{Ag} / \mathrm{AgCl}$ reference electrode. Chronocoulometry was performed at $+0.9 \mathrm{~V}$ versus $\mathrm{Ag} / \mathrm{AgCl}$ until reaching a final charge density of $100 \mathrm{mC} / \mathrm{cm}^{2}$, upon which polymerization was terminated. Freshly polymerized electrodes were dip-rinsed in water and stored dry at room temperature until use.

\section{In Vitro Calibration.}

Electrochemical detection of resting DA was performed via SWV. The SWV waveform scanned from -0.2 to $0.3 \mathrm{~V}$ using a $25 \mathrm{~Hz}$ pulse frequency, $50 \mathrm{mV}$ pulse amplitude, and a 5 $\mathrm{mV}$ step height. SWV scans were applied every $15 \mathrm{~s}$, and potential was held at $0 \mathrm{~V}$ between scans. In vitro DA calibration were performed using freshly prepared, nitrogen-purged DA standard solutions dissolved in artificial cerebrospinal fluid (aCSF, $142 \mathrm{mM} \mathrm{NaCl}, 1.2 \mathrm{mM}$ $\mathrm{CaCl}_{2}, 2.7 \mathrm{mM} \mathrm{KCl}, 1.0 \mathrm{mM} \mathrm{MgCl}, 2.0 \mathrm{mM} \mathrm{NaH}_{2} \mathrm{PO}_{4}, \mathrm{pH}$ 7.4) with and without interfering agents. Electrode sensitivity was determined by the linear slope of the calibration plot relating DA peak current to DA concentration. SWV selectivity for DA at PEDOT/CNTfunctionalized CFEs was determined by performing SWV measurements in a cocktail solution containing ascorbic acid (AA) $(200 \mu \mathrm{M}), 3$,4-dihydroxyphenylacetic acid (DOPAC) $(10 \mu \mathrm{M})$, and uric acid $(10 \mu \mathrm{M}), \mathrm{pH}$ adjusted to 7.4 (all chemicals obtained from SigmaAldrich, St. Louis, MO, U.S.A.). Selectivity was assessed via direct comparison of DA sensitivity in the presence and absence of interfering agents.

\section{In Vivo Procedures.}

The in vivo performance of both PEDOT/CNT-functionalized CFEs and MEAs was determined through acute surgical experiments conducted in dorsal striatum of isoflurane (2\% by volume) anesthetized male Sprague-Dawley rats (350-450 g, Charles River, Wilmington, MA, U.S.A.). All animal procedures were performed according to protocols approved by the University of Pittsburgh Institutional Animal Care and Use Committee. Detailed description of all in vivo methods is provided in the Supporting Information. In 
vivo DA concentration was determined for all in vivo experiments by converting SWV peak current to DA concentration using the precalibration electrode sensitivity.

\section{Data Analysis.}

Each SWV response was first filtered using a zero-phase, forward and reverse (fiach S) lowpass, third-order Butterworth digital filter with the $3 \mathrm{~dB}$ cutoff at 0.25 normalized frequency units $(2.5 \mathrm{~Hz})$ to remove noise. The zero-phase, forward and reverse filter design was chosen to ensure that the SWV peak shape and position were not distorted by the low-pass filter. The DA peak was then isolated from the nonfaradaic background current for each SWV scan by subtracting a modeled polynomial baseline. The fit for the polynomial baseline was determined using a two-step peak extraction method consisting of an iterative peak localization algorithm. First, a linear baseline was initialized with two signal points on either side of a user-selected DA peak maximum voltage ( $0.18 \mathrm{~V}$ for DA). Signal points used to construct the baseline were iteratively updated to produce a final baseline which maximized the subtracted peak amplitude. The resulting linear fit intersects boundary points at either side of the DA peak profile. Portions of the SWV signal existing outside of these bounds closely approximate signals obtained in the absence of DA. The five data points located immediately adjacent to the upper and lower bounds were then modeled using a secondorder polynomial fit. This polynomial fit was extrapolated over the entire SWV potential window and subtracted from the raw SWV response for the purpose of peak extraction.

\section{RESULTS AND DISCUSSION \\ PEDOT/CNT Polymerization of CFEs.}

CFEs undergo successful PEDOT/CNT functionalization upon $+0.9 \mathrm{~V}$ chronocoulometry electrodeposition with a $100 \mathrm{mC} / \mathrm{cm}^{2}$ charge density. PEDOT/CNT deposits as an approximately $1.5 \mu \mathrm{m}$ thick uniform coating on the CFE surface (as observed by SEM diameter comparison between bare and PEDOT/CNT-functionalized CFEs Figure 1, parts a and b). The birdsnest morphology inherent to PEDOT/CNT electropolymerization has been previously shown on both macro- ${ }^{43}$ and microelectrodes ${ }^{44-46}$ accompanied by decreased electrode impedance, increased electrode surface area, and increased electrode capacitance. As predicted, PEDOT/CNT significantly decreases the average overall electrode impedance and significantly increases the capacitance (Figure 1, parts $\mathrm{c}$ and d, $n=5$ ).

The increased electrode surface area, significantly decreased electrode impedance, and significantly increased electrode capacitance following PEDOT/CNT polymerization indicate that PEDOT/CNT-functionalized CFEs exhibit characteristics more similar to a macroelectrode than a microelectrode. In essence, electropolymerization results in a carbonbased macroelectrode compressed into a microelectrode dimension of space. Electrochemistry is a surface phenomenon; as such, the sensitivity for the electrochemical detection of a given analyte increases with the overall electrode surface area. The goal of this study is to develop a fully implantable microelectrode with extreme sensitivity and selectivity for DA such that the selective measurement of trace quantities of resting DA will be possible within the complex brain environment. From a material characteristic standpoint, PEDOT/CNT-functionalized CFEs appear to be an ideal candidate for such a sensor. 


\section{In Vitro Detection of Resting DA at PEDOT/CNT-Functionalized CFEs.}

The performance of PEDOT/CNT-functionalized CFEs as a sensor for the electrochemical detection of resting DA was determined via in vitro calibration experiments performed in aCSF. Xu et al. previously showed that PEDOT/CNT-functionalized carbon paste macroelectrodes are sensitive and selective for resting DA over ascorbic acid interference using differential pulse voltammetry. ${ }^{43}$ Pulsed voltammetric methods, such as differential pulse voltammetry, are designed to directly measure resting analyte concentrations by isolating faradaic current (resulting from redox activity derived from an electroactive analyte) from nonfaradaic charging currents (resulting from the application of a potential waveform). ${ }^{55}$ In this study we update the methodology from that used by Xu et al. by incorporating square wave voltammetry, a pulse voltammetry technique that allows for the isolation of faradic current with improved rate of collection and increased peak current amplitude as compared to differential pulse voltammetry. ${ }^{55-57}$ A schematic of the SWV waveform applied in this study is presented in Figure 2a along with the forward, reverse, and SWV current responses for a representative detection of a $1 \mu \mathrm{M}$ DA standard solution in aCSF (Figure 2b). In addition, a thorough description of SWV is included in the Supporting Information. All SWV parameters were extensively optimized to achieve selective DA detection with maximum peak height and resolution.

The performance of PEDOT/CNT-functionalized CFEs for the electrochemical detection of resting DA was examined by performing in vitro calibration protocols to include varying DA concentrations and common neurochemical interferences. Five individual electrodes were subjected to SWV measurement, first in aCSF, and then in solutions of increasing DA concentration designed to encompass the expected in vivo DA concentration range (100, 250, 500, $1000 \mathrm{nM}$ ). PEDOT/CNT-functionalized CFEs exhibit clear, robust detection of resting DA at each concentration with the average SWV traces (SEM removed for clarity) revealing a single concentration-dependent peak located near $0.18 \mathrm{~V}$ (Figure 2c).

We have developed a highly effective method for establishing a rolling SWV baseline based on second-order polynomial fitting that can be used to selectively isolate current that is DA in origin. This allows for each SWV scan to be considered as a stand-alone entity for the purpose of isolating the faradaic peak current resulting from the electrochemical detection of DA. Each PEDOT/CNT-functionalized CFE produces its own characteristic background current response upon SWV waveform application. This background response is generally stable but can drift and change over time as the electrode/solution interface evolves. Scanning an electrode in aCSF clearly reveals this background current response (Figure 2c, blue). DA detection is observed as the superimposition of a Gaussian faradaic peak centered at $+0.18 \mathrm{~V}$ onto that baseline response (Figure 2c). Accurate DA quantification relies entirely on the ability to properly isolate the DA peak from the baseline SWV current response.

The majority of the DA Gaussian current peak is located between +0.1 and $+0.25 \mathrm{~V}$ (Figure 2c). As a consequence, current corresponding to all other potential regions is of little interest for DA quantification. DA peak isolation presents a challenge in that the baseline SWV current over the +0.1 to $+0.25 \mathrm{~V}$ potential region of interest is nonlinear (Figure 2c, blue). Therefore, simply drawing a straight baseline over the potential region of interest for peak 
isolation results in a gross underestimation of peak height. Accurate isolation of the DA peak requires knowledge of the nonlinear baseline SWV response. We have found that the baseline SWV current response over the +0.1 to $+0.25 \mathrm{~V}$ potential region of interest can be easily fit using a second-order polynomial. The fit only requires the input of a few current values above and below the potential window of interest. The resulting fit equation can then be extrapolated to encompass the entire potential window and serve as a self-contained baseline subtraction for DA peak isolation. The upper and lower current input bounds for the second-order polynomial are determined using an iterative peak localization algorithm described in the Materials and Methods section.

The fit for the polynomial baseline was determined using a two-step peak extraction method consisting of an iterative peak localization algorithm. First, a linear baseline was initialized with two signal points on either side of a user-selected DA peak maximum voltage $(0.18 \mathrm{~V}$ for DA). Signal points used to construct the baseline were iteratively updated to produce a final baseline which maximized the subtracted peak amplitude. The resulting linear fit intersects boundary points at either side of the DA peak profile. Portions of the SWV signal existing outside of these bounds closely approximate signals obtained in the absence of DA. The five data points located immediately adjacent to the upper and lower bounds were then modeled using a second-order polynomial fit. This polynomial fit was extrapolated over the entire SWV potential window and subtracted from the raw SWV response for the purpose of peak extraction.

The effectiveness of the second-order polynomial fit for modeling the SWV baseline current response within the potential region of interest $(+0.1$ to $+0.25 \mathrm{~V})$ is clearly displayed by modeling the average SWV current responses (previously presented in Figure 2c) for aCSF (Figure 2d, blue) and $250 \mathrm{nM}$ DA (Figure 2d, green). The second-order polynomial produces a calculated baseline that is almost identical over the +0.1 to $+0.25 \mathrm{~V}$ potential region of interest to the raw SWV current response collected in aCSF (Figure 2d, top dashed line). The second-order polynomial remains functional following the introduction of $250 \mathrm{nM}$ DA (Figure 2d, bottom dashed line), drawing an adequate nonlinear baseline below the DA peak centered at $0.18 \mathrm{~V}$. The average SWV responses $( \pm \mathrm{SEM})$ for each DA concentration following polynomial subtraction (Figure 2e) reveal clear Gaussian peaks as predicted by theory. ${ }^{55-57}$ This Gaussian peak shape serves as evidence supporting the use of a polynomial baseline subtraction method for faradaic DA peak isolation. Further validation supporting the use of this polynomial baseline subtraction method was provided through the comparison of DA calibration plots.

Electrode sensitivity for the electrochemical detection of resting DA was defined as the linear slope of the calibration plot relating the background-subtracted SWV peak current to DA standard concentration. The average $( \pm$ SEM) DA calibration plot, generated by plotting the average maximum peak current from five individual PEDOT/CNT-functionalized CFEs following polynomial background subtraction (Figure 2f, red, $n=5$ ), is highly linear $\left(r^{2}>\right.$ 0.99 ) with a $90 \pm 7 \mathrm{nA} / \mu \mathrm{M}$ sensitivity for resting DA detection. The average ( \pm SEM) calibration plot for bare CFEs following polynomial background subtraction is also highly linear (Figure $2 \mathrm{f}$, black, $n=3$ ) but exhibits only a $0.21 \pm 0.08 \mathrm{nA} / \mu \mathrm{M}$ sensitivity. The average sensitivities reported were determined by averaging the individual sensitivities for 
each separate electrode. The linear regression fit on the average calibration plot (Figure 2f) is presented for the purpose of illustrating average sensitivity, not to quantify average sensitivity. This explains the slight deviation from the reported average sensitivity and the slope produced by the average calibration plot (Figure 2f). Direct comparison of DA sensitivity at bare and PEDOT/CNT-functionalized CFEs reveals that PEDOT/CNT significantly increases electrode sensitivity for DA by 422 -fold (one-way ANOVA, $p<$ 0.05 ). These results unequivocally confirm the effectiveness of SWV measurement at PEDOT/CNT-functionalized CFEs for the direct detection of resting DA. The relatively poor performance of bare CFEs for the in vitro detection of resting DA using SWV as compared to previous studies using DNPV can be clearly explained by differences in electrode preparation. Studies using DNPV employed CFEs composed of a larger exposed carbon fiber (12 $\mu \mathrm{m}$ diameter, $700 \mu \mathrm{m}$ length) that had undergone extensive electrochemical pretreatment (2.8 V maximum voltage) to increase electrode sensitivity.

Validation of the second-order polynomial baseline subtraction method for isolating the faradaic DA peak current was performed by comparing the previously described average $( \pm$ SEM) PEDOT/CNT calibration plot constructed following polynomial baseline subtraction (Figure $2 \mathrm{f}$, red) to the average $( \pm \mathrm{SEM})$ calibration plot constructed from the same PEDOT/CNT-functionalized CFEs following traditional aCSF baseline subtraction (Figure 2f, blue). The average calibration response for DA following aCSF subtraction is highly linear $\left(r^{2}>0.99\right)$ with a $108 \pm 9 \mathrm{nA} / \mu \mathrm{M}$ sensitivity for resting DA detection. The average sensitivity for DA detection obtained by aCSF background subtraction is not significantly different from the $90 \pm 7 \mathrm{nA} / \mu \mathrm{M}$ sensitivity for resting DA detection obtained using the previously described polynomial background subtraction method (one-way ANOVA, $p>0.05$ ). Although not significant, it should be noted that the DA peaks obtained by polynomial background subtraction exhibit slightly smaller peak heights than aCSF subtraction. The average sensitivity for DA following polynomial subtraction is $83 \%$ of the sensitivity produced by aCSF subtraction. This indicates that the second-order polynomial fit modeled in the presence of $250 \mathrm{nM}$ DA is slightly shallower than for true baseline exhibited by aCSF alone. Regardless of the 17\% signal loss, the polynomial baseline subtraction method produces clear, quantifiable Gaussian peaks for the electrochemical detection of resting DA. It should also be noted that the signal loss associated with incorporating polynomial baseline subtraction will not introduce additional error into the quantification as long as it is used at each step of the experimental process (in vivo collection, in vitro calibration, etc.). The same $\sim 17 \%$ error will be propagated at each step of the process.

SWV detection at PEDOT/CNT-functionalized CFEs exhibit higher sensitivity for resting DA than FSCAV $\left(81 \pm 11 \mathrm{nA} / \mu \mathrm{M}^{34}\right)$, charged balanced waveform FSCV $(85.4 \pm 14.3 \mathrm{nA} /$ $\mu \mathrm{M}^{32}$ ), or convolution-based FSCV nonfaradaic current removal $\left(10.7 \pm 0.3 \mathrm{nA} / \mu \mathrm{M}^{38}\right)$. The sensitivity of electrochemically pretreated CFEs for resting DA using DNPV is not clearly defined in the literature. The average lower limit of detection for DA using PEDOT/CNTfunctionalized CFEs, defined at 3 times the standard deviation of the noise, was determined to be $2.03 \pm 0.09 \mathrm{nM}$, which is far below in vivo basal DA concentrations reported by Heien and Wightman's groups $(40-100 \mathrm{nM}) .{ }^{35,38}$ 
It is important to note that the PEDOT/CNT nanocomposite coating is absolutely essential for successful DA detection. PEDOT/CNT deposits as a high surface area carbon-based "birds-nest" morphology (Figure 1b). Acid-functionalized CNTs are negatively charged, ${ }^{58,59}$ whereas DA is positively charged at physiological $\mathrm{pH}$ (7.4). Incorporation of negatively charged components onto the surface of the electrode will facilitate DA adsorption. ${ }^{51}$ Since electrochemistry is a surface phenomenon, increasing the electrode surface area with a material capable of selectively facilitating DA adsorption will increase sensitivity by concentrating DA onto the electrode surface. It should be noted that bare CFEs also exhibit a negatively charged surface due to the presence of oxygen-containing functional groups but still exhibit poor sensitivity for resting DA via SWV detection (Figure 2e). This suggests that the PEDOT/CNT-mediated increase in sensitivity requires both an increase in negative charge and effective surface area.

Due to the highly porous and adsorptive nature of PEDOT/CNT coatings there is a concern that DA diffusion in and out of the coating will be restricted such that the temporal response of the electrode will be slowed and DA may actually become trapped within the coating. This concern has been alleviated by incorporating an $11 \mathrm{~s} 0 \mathrm{~V}(\mathrm{vs} \mathrm{Ag} / \mathrm{AgCl})$ hold into the SWV waveform. DA is able to diffuse $123 \mu \mathrm{m}$ during the $11 \mathrm{~s}$ hold period $(\sqrt{2 D t}$, where $D=$ $\left.6.9 \mathrm{~cm}^{2} / \mathrm{s}^{60}\right)$. This time is more than sufficient for DA within the approximately $1.5 \mu \mathrm{m}$ thick $\mathrm{PEDOT} / \mathrm{CNT}$ coating to reach equilibrium with the surrounding environment. In addition, 0 $\mathrm{V}$ versus $\mathrm{Ag} / \mathrm{AgCl}$ is a potential where DAoQ is reduced back to DA at physiological $\mathrm{pH}$. This ensures that DA located within and directly surrounding the electrode coating is reduced prior to subsequent detection. The presence of retained DA within the PEDOT/CNT coating is not likely to affect the ability of PEDOT/CNT-functionalized CFEs to accurately quantify in vivo basal DA, as the same retention would occur in both in vivo and in vitro settings. As such, any signal enhancement due to the retention of DA within the PEDOT/CNT coating will be reflected in the in vitro calibration. Incorporation of the $11 \mathrm{~s}$ hold into the SWV waveform results in an overall temporal resolution of $15 \mathrm{~s}$ for the detection of resting DA.

It is imperative that SWV detection be selective over common neurochemical interferences found throughout the complex brain environment. To assess selectivity, DA calibrations were performed at PEDOT/CNT-functionalized CFEs in aCSF containing a cocktail of common neurochemical interferences prepared to reflect expected physiological concentrations in the rat dorsal striatum. The interference cocktail consisted of $200 \mu \mathrm{M}$ AA, $10 \mu \mathrm{M}$ uric acid (UA), and $10 \mu \mathrm{M}$ DOPAC $\mathrm{pH}$ adjusted to 7.4. The average ( $n=5$, SEM removed for clarity) baseline SWV response in the presence of the contaminant cocktail (Figure 3a, blue) showed an overall increase in the background current (as compared to aCSF alone, Figure 2c, blue) but no apparent faradaic current peaks within the -0.2 to $0.3 \mathrm{~V}$ potential window. Introduction of increasing concentrations of DA results in the formation of a clear faradaic peak located at $0.18 \mathrm{~V}$ (Figure 3a). This peak location is in complete agreement with calibrations performed in aCSF alone (Figure 2c).

The faradaic DA peak can again be isolated using the previously described polynomial background subtraction procedure. The effectiveness of the second-order polynomial fit for modeling the SWV baseline current response within the potential region of interest $(+0.1$ to 
$+0.25 \mathrm{~V}$ ) in the presence of the AA, UA, DOPAC interference cocktail is clearly displayed by modeling the average SWV current responses (previously presented in Figure 3a) for AA, UA, and DOPAC alone (Figure 3b, blue) and AA, UA, DOPAC plus $250 \mathrm{nM}$ DA (Figure 3b, green). Similar to before, the second-order polynomial produces a highly correlation fit over the +0.1 to $+0.25 \mathrm{~V}$ potential region of interest to the average SWV current response collected in the interference cocktail alone (Figure 3b, top dashed line) and also draws a suitable nonlinear baseline under the DA peak for the AA, UA, DOPAC plus $250 \mathrm{nM}$ DA average SWV response (Figure 3b, bottom dashed line). The subtraction of the polynomialderived baseline from the raw SWV response results in a clear Gaussian-shaped peak centered around $0.18 \mathrm{~V}$ for DA containing standards and no discernible peak for the interference cocktail alone. The average $( \pm$ SEM) DA calibration plot for PEDOT/CNTfunctionalized CFEs following polynomial background subtraction in the presence of the AA, UA, DOPAC interference cocktail (Figure 3c, $n=5$ ) is highly linear with a $93 \pm 15 \mathrm{nA}$ / $\mu \mathrm{M}$ sensitivity for resting DA detection. This sensitivity is not significantly different than the sensitivity obtained by DA calibration in aCSF alone. This clearly indicates that the combined usage of PEDOT/CNT-functionalized electrodes, SWV detection, and polynomial baseline subtraction allows for the sensitive and selective detection of DA.

It is notable that PEDOT/CNT-functionalized CFEs provide such excellent selectivity over such a range of neurochemical interferences, most notably DOPAC and AA. DOPAC and AA have similar standard reduction potentials to $\mathrm{DA}^{61}$ and are present in the brain at concentrations that are orders of magnitude higher than DA. ${ }^{62,63}$ As such, electrochemical measurement of submicromolar DA concentrations in the presence of DOPAC and AA has proven challenging. PEDOT/CNT owes its excellent selectivity over DOPAC and AA to electrostatic repulsion. ${ }^{43}$ As previously mentioned, PEDOT/CNT nanocomposite coatings incorporate negatively charged acid-functionalized CNTs 58,59 DOPAC and AA also carry a negative charge at physiological $\mathrm{pH}$ (7.4). Electrostatic repulsion prevents DOPAC, AA, and any other negatively charged interfering molecule from approaching the electrode surface and contributing to the SWV current. In this aspect, PEDOT/CNT is producing a similar effect to Nafion, a negatively charged coating typically deposited onto the surface of CFEs to provide electrostatic separation of positively charged neurochemicals over negatively charged interferences, such as AA and DOPAC. ${ }^{35}$

\section{In Vivo Measurement of Basal DA at PEDOT/CNT-Functionalized CFEs.}

The in vivo performance of the PEDOT/CNT-functionalized CFEs for the detection of basal DA was determined through direct implantation into the dorsal striatum of isofluraneanesthetized rats. The SWV waveform (detailed previously) was applied immediately upon finalizing electrode placement and continuously repeated over a period of $90 \mathrm{~min}$. Following $30 \mathrm{~min}$ of baseline SWV collection, $n=3$ individual rats received a single $20 \mathrm{mg} / \mathrm{kg}$ ip injection of the competitive dopamine transporter inhibitor nomifensine and $n=3$ individual rats received a single ip saline injection (vehicle). Nomifensine has previously been shown to increase basal DA. ${ }^{32,64}$ Application of the SWV waveform (accompanied by polynomial baseline subtraction) produced a clear DA peak (Figure 4a, black) which was increased following $20 \mathrm{mg} / \mathrm{kg}$ ip nomifensine (Figure $4 \mathrm{a}$, red). The average $(n=3$, polynomial baseline-subtracted) SWV response collected in the non-DA-rich forelimb region of the 
primary somatosensory cortex (S1FL, Figure 4a, green) does not exhibit a discernible peak in the potential region of interest for DA. This clear absence of a DA peak in the non-DAcontaining S1FL provides clear support that the SWV peak recorded in the dorsal striatum is DA in origin. In vivo SWV peak current was converted to DA concentration using preimplantation in vitro electrode calibration. Preimplantation in vitro electrode calibration was chosen for the conversion of in vivo SWV current to DA concentration due to the observed stability of the in vivo current response. This stability is clearly evident in the lack of drift observed at PEDOT/CNT-functionalized CFEs during saline controls (Figure 4). Stability is observed immediately upon probe implantation (less than $1 \mathrm{~min}$ ) without requiring electrochemical in vivo stabilization. Postexplantation in vitro calibration was not used for the assignment of in vivo DA concentration due to a $71 \%$ decrease in in vitro DA sensitivity observed following probe explantation. This decrease in sensitivity is likely due to the encapsulation of the PEDOT/CNT-functionalized CFE with biological matter (blood) during the explantation process (Figure S2). This encapsulation is unlikely to occur during implantation as careful surgical technique was employed to ensure that implantation sites were free of blood. Additionally, encapsulation is unlikely during the 90 min of implantation because the acute time frame is shorter than the time required for glial encapsulation. ${ }^{65}$

The average basal DA concentration in the dorsal striatum was quantified to be $82 \pm 6 \mathrm{nM}$ (Figure $4 \mathrm{~b}, n=6$ ). DA concentration in the dorsal striatum is comparable to measurements obtained in the nucleus accumbens of mice $(90 \pm 9 \mathrm{nM})$ and rats $(41 \pm 13 \mathrm{nM})$ by FSCAV and convolution-based FSCV, respectively. ${ }^{35,38}$ This measurement is also comparable to the $120 \pm 18 \mathrm{nM}$ basal concentration reported in the rat striatum using $\mathrm{m}-\mathrm{CSWV}^{39}$ but an order of magnitude less than the measurement provided by FSCV experiments involving intracranial infusion of kynurenate $(>2.6 \mu \mathrm{M})^{11}$ and much higher than the $26 \pm 8 \mathrm{nM}$ concentration obtained using DNPV in pargyline-pretreated striatum. The immediate stability of the SWV peak current (Figure 4) indicates that CFE implantation does not result in locally stimulated DA release. This is in agreement with other studies where basal DA was determined at acutely implanted CFEs. ${ }^{35,37,38,40}$

Following nomifensine injection at $t=30 \mathrm{~min}$, basal DA began to increase after approximately $5 \mathrm{~min}$ and continued to increase until reaching a maximum DA concentration $207 \pm 16 \mathrm{nM}$ at $28 \pm 2 \mathrm{~min}$ following injection (Figure $4 \mathrm{~b}$, red, $n=3$ ). The average DA response in saline control animals remained steady following ip injection (Figure 4b, black, $n=3$ ). This nomifensine-induced DA increase is in complete agreement with previous measurements of tonic DA using both electrochemistry ${ }^{32}$ and microdialysis. ${ }^{66}$ We recognize the disparity in basal DA concentration reported by different techniques. At this point, it is difficult to know which estimate is correct, though it is notable that the change in basal DA induced by ip nomifensine injection is similar for each method, which adds legitimacy to each reported method. We are confident in our reproducibly measured $82 \pm 6 \mathrm{nM}$ basal DA concentration due to the presence of a clear, nomifensine-dependent DA peak observed during all in vivo measurements (Figure 4a).

All in vivo basal level responses showed substantial current fluctuation over time. While this appears at first to be random noise associated with the electrochemical detection, multiple attempts at applying a low-pass filter to current versus time plots failed to remove this 
feature entirely. As such, it is entirely possible that this observed fluctuation is indeed physiological in nature and corresponds to small fluctuations in local basal DA concentration. Further testing will be required to validate this theory. In all, these in vivo findings unequivocally prove the effectiveness of performing SWV at PEDOT/CNTfunctionalized CFEs for the direct in vivo measurement of basal DA.

\section{DA Detection at PEDOT/CNT-Functionalized MEAs.}

We have clearly established that PEDOT/CNT-functionalized CFEs are sensitive and selective for the electrochemical detection of resting DA via SWV both in vitro and in vivo. In addition, we have established that incorporation of the PEDOT/CNT nanocomposite coating is absolutely essential to that detection. One of the most attractive features of conductive polymers is that they can be deposited onto a wide variety of conductive substrates. As electrochemistry is a surface phenomenon, it is reasonable to believe that this basal DA detection methodology can be transferred to another electrode substrate by selectively depositing the PEDOT/CNT nanocomposite coating. Silicon-based MEAs developed for electrophysiological recordings are capable of measuring neural activity from multiple sites across different depths and widths of brain tissue with high-quality, single-cell resolution. ${ }^{67}$ However, MEAs are not as widely used for neurochemical sensing because conventional electrode site materials ( $\mathrm{Au}, \mathrm{Pt}, \mathrm{Ir}$ ) are not sensitive and selective for electrochemical detection ${ }^{68}$ without substantial electrode modification. ${ }^{69-72}$ Here we aim to convert individual $1200 \mu \mathrm{m}^{2}$ gold electrode sites along a silicon MEA into functional basal DA sensing electrodes.

PEDOT/CNT-functionalized MEAs perform in a strikingly similar manner to PEDOT/CNTfunctionalized CFEs. Individual gold electrode sites undergo selective, uniform PEDOT/CNT coating upon $+0.9 \mathrm{~V}$ chronocoulometry electropolymerization with a 100 $\mathrm{mC} / \mathrm{cm}^{2}$ charge density cutoff. The average $(n=5)$ in vitro DA calibration response at PEDOT/CNT-functionalized MEAs produces a clear, concentration-dependent Gaussian DA peak (Figure $5 \mathrm{~b}$, inset) centered around $+0.18 \mathrm{~V}$ superimposed onto the baseline SWV current response (Figure 5b). Comparison of the average peak current to DA concentration reveals excellent linearity and a $0.0147 \pm 0.0005 \mathrm{nA} / \mu \mathrm{M}$ average sensitivity (Figure $5 \mathrm{~b}$ ). The sensitivity of PEDOT/CNT-functionalized MEAs for resting DA detection is approximately $10 \times$ smaller than that of PEDOT/CNT-functionalized CFEs. This is likely due to the approximately $10 \times$ difference in surface area present between the two electrode substrates prior to PEDOT/CNT coating. Incorporation of PEDOT/CNT onto the electrode surface is again absolutely essential for DA detection. In fact, bare gold MEA electrode sites are completely insensitive for DA detection via SWV (Figure 5b inset). On average ( $n=5$ ), gold MEA electrodes do not display a concentration-dependent SWV peak upon increasing DA concentration. This is apparent by the appearance of nonlinear, negative $-0.024 \pm 0.029 \mathrm{pA} /$ $\mu \mathrm{M}$ relationship between current and DA concentration. The ability to convert a totally DAinsensitive electrode substrate into a highly sensitive DA sensing electrode unequivocally reveals the power and versatility of PEDOT/CNT nanocomposite coatings. These findings suggest that a variety of conductive substrates, whether inherently sensitive for the electrochemical detection of DA (i.e., CFEs) or not (i.e., gold MEA electrodes), can be converted into DA sensing electrodes by simply depositing PEDOT/CNT. 
Motivated by the excellent in vitro performance of PEDOT/CNT-functionalized MEAs, we performed a proof-of-principle in vivo experiment in an isoflurane-anesthetized rat designed to show the spatial resolution afforded by measuring basal DA at a silicon-based MEA. We first polymerized three individual gold electrode sites along the MEA spanning a $2.8 \mathrm{~mm}$ vertical distance with the middle electrode located $1.2 \mathrm{~mm}$ from the ventral-most electrode. The MEA was positioned above the dorsal striatum $(2.5 \mathrm{~mm}$ lateral and $0.43 \mathrm{~mm}$ anterior to bregma) and lowered to a final position $5.4 \mathrm{~mm}$ below the cortical surface (see the drawing in Figure 5c) such that two of the PEDOT/CNT-functionalized electrodes sites were located in the DA-rich dorsal striatum (Figure 5c, green and black) while the last functionalized electrode site was located in the non-DA-rich S1FL (Figure 5c, red). Upon finalizing MEA implantation, the electrical circuit was completed with the $\mathrm{Ag} / \mathrm{AgCl}$ reference and bone screw counter electrode as detailed previously. Once the electrodes were in place, the SWV waveform was repeatedly applied to each individual electrode in succession over a period of $90 \mathrm{~min}$. We mimicked the previous in vivo experimental design by injecting $20 \mathrm{mg} / \mathrm{kg}$ ip nomifensine following $30 \mathrm{~min}$ of baseline collection (Figure 5d, blue dashed vertical line). The two PEDOT/CNT-functionalized gold MEA electrode sites located within the dorsal striatum (green and black) each exhibited clear DA detection as well as a site-specific corresponding increase in basal DA following ip nomifensine administration (Figure 5d). In all, both responses are similar to those detected at PEDOT/CNT-functionalized CFEs (Figure 4 b). Conversely, the electrode located within the non-DA-rich S1FL does not detect a clear DA peak, nor does it detect a current change upon ip nomifensine administration. This proof-of-principle in vivo experiment clearly shows the power of incorporating the DAsensitive PEDOT/CNT coating onto multiple electrode sites along an MEA substrate. Recording from spatially isolated electrode sites located both within and between brain regions can provide a depth of information regarding the chemistry of multiple specific neuron groupings. Overall, the time-correlated, multisite quantification of basal DA in the brain represents a substantial advancement in DA sensing technology.

\section{CONCLUSIONS}

This study detailed development in the direct quantification of in vivo resting DA based on SWV detection at individual PEDOT/CNT-functionalized microelectrodes. This technology relies interdependently on two major innovations, PEDOT/CNT coating and SWV detection. $\mathrm{PEDOT/CNT}$ is a highly conductive nanocomposite coating that exhibits extreme sensitivity for DA, while SWV is an electrochemical technique capable of directly measuring resting levels due to the selective elimination of nonfaradaic background charging currents. Furthermore, electrodeposition of PEDOT/CNT allows for the conversion of widely used electrode materials (CFEs, MEAs, etc.) into highly sensitive and selective DA sensors. SWV detection of resting DA absolutely requires PEDOT/CNT functionalization as bare electrodes exhibit little-to-no sensitivity for DA. PEDOT/CNT-functionalized sensors are capable of directly quantifying resting DA in the rat dorsal striatum with high spatial and temporal resolution. Multisite resting DA detection could serve to tremendously improve our understanding of DA function in both the healthy and pathological brain. We hope to further expand this technology to allow for chronic multisite DA measurement as well as simultaneous electrophysiological recording from MEAs. 


\section{Supplementary Material}

Refer to Web version on PubMed Central for supplementary material.

\section{ACKNOWLEDGMENTS}

We would like to thank Drs. Adrian Michael and Stephen Weber at the University of Pittsburgh for their valuable insight regarding this work. We would also like to thank Moriah McGuier and Julian Metro from Saint Vincent College for their assistance with the interference testing. This work was supported by NIH (Grants R01NS062019, R01NS089688, R21DA049592, and R21DA043817).

\section{REFERENCES}

(1). Brooks DJ J. Neural Transm 2001, 108, 1283-1298. [PubMed: 11768627]

(2). Hull EM; Lorrain DS; Du J; Matuszewich L; Lumley LA; Putnam SK; Moses J Behav. Brain Res 1999, 105, 105-116. [PubMed: 10553694]

(3). Urban NL; Slifstein M; Meda S; Xu X; Ayoub R; Medina O; Pearlson G; Krystal J; Abi-Dargham A Psychopharmacology (Berl.) 2012, 221, 67-77. [PubMed: 22052081]

(4). Floresco SB; West AR; Ash B; Moore H; Grace AA Nat. Neurosci 2003, 6, 968-973. [PubMed: 12897785]

(5). Grace AA Neuroscience 1991, 41, 1-24. [PubMed: 1676137]

(6). Grace AA Nat. Rev. Neurosci 2016, 17, 524-532. [PubMed: 27256556]

(7). Schultz W Annu. Rev. Neurosci 2007, 30, 259-288. [PubMed: 17600522]

(8). Schultz WJ Neurophysiol. 1998, 80, 1-27.

(9). Robinson DL; Venton BJ; Heien MLAV; Wightman RM Clin. Chem 2003, 49, 1763-1773. [PubMed: 14500617]

(10). Wightman RM; May LJ; Michael AC Anal. Chem 1988, 60, 769A-779A.

(11). Borland LM; Michael AC J. Neurochem 2004, 91, 220-229. [PubMed: 15379902]

(12). Carboni E; Imperato A; Perezzani L; Dichiara G Neuroscience 1989, 28, 653-661. [PubMed: 2710338]

(13). Carboni E; Spielewoy C; Vacca C; Nosten-Bertrand M; Giros B; Di Chiara GJ Neurosci. 2001, $21, \mathrm{RC} 141$.

(14). Di Chiara G; Carboni E; Morelli M; Cozzolino A; Tanda GL; Pinna A; Russi G; Consolo S Neuroscience 1993, 55, 451-456. [PubMed: 8377936]

(15). Pontieri FE; Tanda G; Di Chiara G Proc. Natl. Acad. Sci. U. S. A 1995, 92, 12304-12308. [PubMed: 8618890]

(16). Auclair A; Cotecchia S; Glowinski J; Tassin J-PJ Neurosci. 2002, 22, 9150-9154.

(17). Rassoulpour A; Wu H-Q; Ferrè S; Schwarcz R J. Neurochem 2005, 93, 762-765. [PubMed: 15836634]

(18). Wu H-Q; Rassoulpour A; Schwarcz RJ Neural Transm. 2007, 114, 33-41.

(19). Chen NNH; Lai Y-J; Pan WHT Neurosci. Lett 1997, 225, 197-200. [PubMed: 9147404]

(20). Tang A; Bungay PM; Gonzales RA J. Neurosci. Methods 2003, 126, 1-11. [PubMed: 12788497]

(21). Martin-Fardon R; Sandillon F; Thibault J; Privat A; Vignon JJ Neurosci. Methods 1997, 72, 123 135.

(22). Parsons LH; Justice JB Jr J. Neurochem 1992, 58, 212-218. [PubMed: 1727431]

(23). Watson CJ; Venton BJ; Kennedy RT Anal. Chem 2006, 78, 1391-1399. [PubMed: 16570388]

(24). Borland LM; Shi GY; Yang H; Michael AC J. Neurosci. Methods 2005, 146, 149-158. [PubMed: 15975664]

(25). Jaquins-Gerstl A; Michael AC J. Neurosci. Methods 2009, 183, 127-135. [PubMed: 19559724]

(26). Jaquins-Gerstl A; Shu Z; Zhang J; Liu YS; Weber SG; Michael AC Anal. Chem 2011, 83, 76627667. [PubMed: 21859125] 
(27). Nesbitt KM; Jaquins-Gerstl A; Skoda EM; Wipf P; Michael AC Anal. Chem 2013, 85, 81738179. [PubMed: 23927692]

(28). Nesbitt KM; Varner EL; Jaquins-Gerstl A; Michael AC ACS Chem. Neurosci 2015, 6, 163-173. [PubMed: 25491242]

(29). Varner EL; Jaquins-Gerstl A; Michael AC ACS Chem. Neurosci 2016, 7, 728-736. [PubMed: 27003503]

(30). Bassareo V; Cucca F; Frau R; Di Chiara G Behav. Brain Res 2015, 287, 200-206. [PubMed: 25827930]

(31). Bungay PM; Newton-Vinson P; Isele W; Garris PA; Justice JB Jr J. Neurochem 2003, 86, 932946. [PubMed: 12887691]

(32). Oh Y; Park C; Kim DH; Shin H; Kang YM; DeWaele M; Lee J; Min H-K; Blaha CD; Bennet KE; Kim IY; Lee KH; Jang DP Anal. Chem 2016, 88, 10962-10970. [PubMed: 27774784]

(33). Atcherley CW; Laude ND; Monroe EB; Wood KM; Hashemi P; Heien ML ACS Chem. Neurosci 2015, 6, 1509-1516. [PubMed: 25062330]

(34). Atcherley CW; Laude ND; Parent KL; Heien ML Langmuir 2013, 29, 14885-14892. [PubMed: 24245864]

(35). Atcherley CW; Wood KM; Parent KL; Hashemi P; Heien ML Chem. Commun 2015, 51, 22352238.

(36). Burrell MH; Atcherley CW; Heien ML; Lipski J ACS Chem. Neurosci 2015, 6, 1802-1812. [PubMed: 26322962]

(37). Johnson JA; Hobbs CN; Wightman RM Anal. Chem 2017, 89, 6166-6174. [PubMed: 28488873]

(38). Johnson JA; Rodeberg NT; Wightman RM Anal. Chem 2018, 90, 7181-7189. [PubMed: 29806450]

(39). Oh Y; Heien ML; Park C; Kang YM; Kim J; Boschen SL; Shin H; Cho HU; Blaha CD; Bennet KE; Lee HK; Jung SJ; Kim IY; Lee KH; Jang DP Biosens. Bioelectron 2018, 121, 174-182. [PubMed: 30218925]

(40). Gonon FG; Buda MJ Neuroscience 1985, 14, 765-774. [PubMed: 2986044]

(41). Suaud-Chagny MF; Chergui K; Chouvet G; Gonon F Neuroscience 1992, 49, 63-72. [PubMed: 1357587]

(42). Gonzalez-Mora JL; Guadalupe T; Fumero B; Mas MJ Neurosci. Methods 1991, 39, 231-244.

(43). Xu G; Li B; Cui XT; Ling L; Luo X Sens. Actuators, B 2013, 188, 405-410.

(44). Alba N; Du Z; Catt K; Kozai T; Cui X Biosensors 2015, 5, 618. [PubMed: 26473938]

(45). Kozai TDY; Catt K; Du Z; Na K; Srivannavit O; Haque R.-u. M.; Seymour J; Wise KD; Yoon E; Cui XT IEEE Trans. Biomed. Eng 2016, 63, 111-119. [PubMed: 26087481]

(46). Luo X; Weaver CL; Zhou DD; Greenberg R; Cui XT Biomaterials 2011, 32, 5551-5557. [PubMed: 21601278]

(47). Kolarcik CL; Catt K; Rost E; Albrecht IN; Bourbeau D; Du Z; Kozai TDY; Luo X; Weber DJ; Cui XT J. Neural Eng 2015, 12, 016008. [PubMed: 25485675]

(48). Taylor IM; Ilitchev AI; Michael AC ACS Chem. Neurosci 2013, 4, 870-878. [PubMed: 23600442]

(49). Taylor IM; Jaquins-Gerstl A; Sesack SR; Michael AC J. Neurochem 2012, 122, 283-294. [PubMed: 22548305]

(50). Taylor IM; Nesbitt KM; Walters SH; Varner EL; Shu Z; Bartlow KM; Jaquins-Gerstl AS; Michael AC J. Neurochem 2015, 133, 522-531. [PubMed: 25683259]

(51). Taylor IM; Robbins EM; Catt KA; Cody PA; Happe CL; Cui XT Biosens. Bioelectron 2017, 89, 400-410. [PubMed: 27268013]

(52). Taylor IM; Du Z; Bigelow ET; Eles JR; Horner AR; Catt KA; Weber SG; Jamieson BG; Cui XT J. Mater. Chem. B 2017, 5, 2445. [PubMed: 28729901]

(53). Du ZJ; Bi GQ; Cui XT Adv. Funct. Mater 2018, 28, 1703988. [PubMed: 30467460]

(54). Weaver CL; Li H; Luo X; Cui XT J. Mater. Chem. B 2014, 2, 5209-5219. [PubMed: 32261663]

(55). Bard AJ; Faulkner LR Electrochemical Methods: Fundamentals and Applications, 2nd ed.; John Wiley \& Sons, Inc.: Hoboken, NJ, 2001. 
(56). Ramaley L; Krause MS Anal. Chem 1969, 41, 1362-1365.

(57). Osteryoung JG; Osteryoung RA Anal. Chem 1985, 57, 101-110.

(58). Zhang J; Zou H; Qing Q; Yang Y; Li Q; Liu Z; Guo X; Du ZJ Phys. Chem. B 2003, 107, 3712 3718.

(59). Chen J; Hamon MA; Hu H; Chen Y; Rao AM; Eklund PC; Haddon RC Science 1998, $282,95$. [PubMed: 9756485]

(60). Sykova E; Nicholson C Physiol. Rev 2008, 88, 1277-1340. [PubMed: 18923183]

(61). Schmidt AC; Wang X; Zhu Y; Sombers LA ACS Nano 2013, 7, 7864-7873. [PubMed: 23941323]

(62). Venton BJ; Troyer KP; Wightman RM Anal. Chem 2002, 74, 539-546. [PubMed: 11838672]

(63). Smith AD; Olson RJ; Justice JB Jr J. Neurosci. Methods 1992, 44, 33-41. [PubMed: 1279321]

(64). Zetterström T; Sharp T; Collin AK; Ungerstedt U Eur. J. Pharmacol 1988, 148, 327-334. [PubMed: 3384000]

(65). Kozai TDY; Jaquins-Gerstl AS; Vazquez AL; Michael AC; Cui XT ACS Chem. Neurosci 2015, 6, 48-67. [PubMed: 25546652]

(66). Gu H; Varner EL; Groskreutz SR; Michael AC; Weber SG Anal. Chem 2015, 87, 6088-6094. [PubMed: 25970591]

(67). Obien MEJ; Deligkaris K; Bullmann T; Bakkum DJ; Frey U Front. Neurosci 2015, 8, 423. [PubMed: 25610364]

(68). Johnson MD; Franklin RK; Gibson MD; Brown RB; Kipke DR J. Neurosci. Methods 2008, 174, 62-70. [PubMed: 18692090]

(69). Vasylieva N; Marinesco S; Barbier D; Sabac A Biosens. Bioelectron 2015, 72, 148-155. [PubMed: 25978443]

(70). Lourenço CF; Ledo A; Laranjinha J; Gerhardt GA; Barbosa RM Sens. Actuators, B 2016, 237, 298-307.

(71). Rutherford EC; Pomerleau F; Huettl P; Strömberg I; Gerhardt GA J. Neurochem 2007, 102, 712722. [PubMed: 17630982]

(72). Tseng TTC; Monbouquette HG J. Electroanal. Chem 2012, 682, 141-146. 

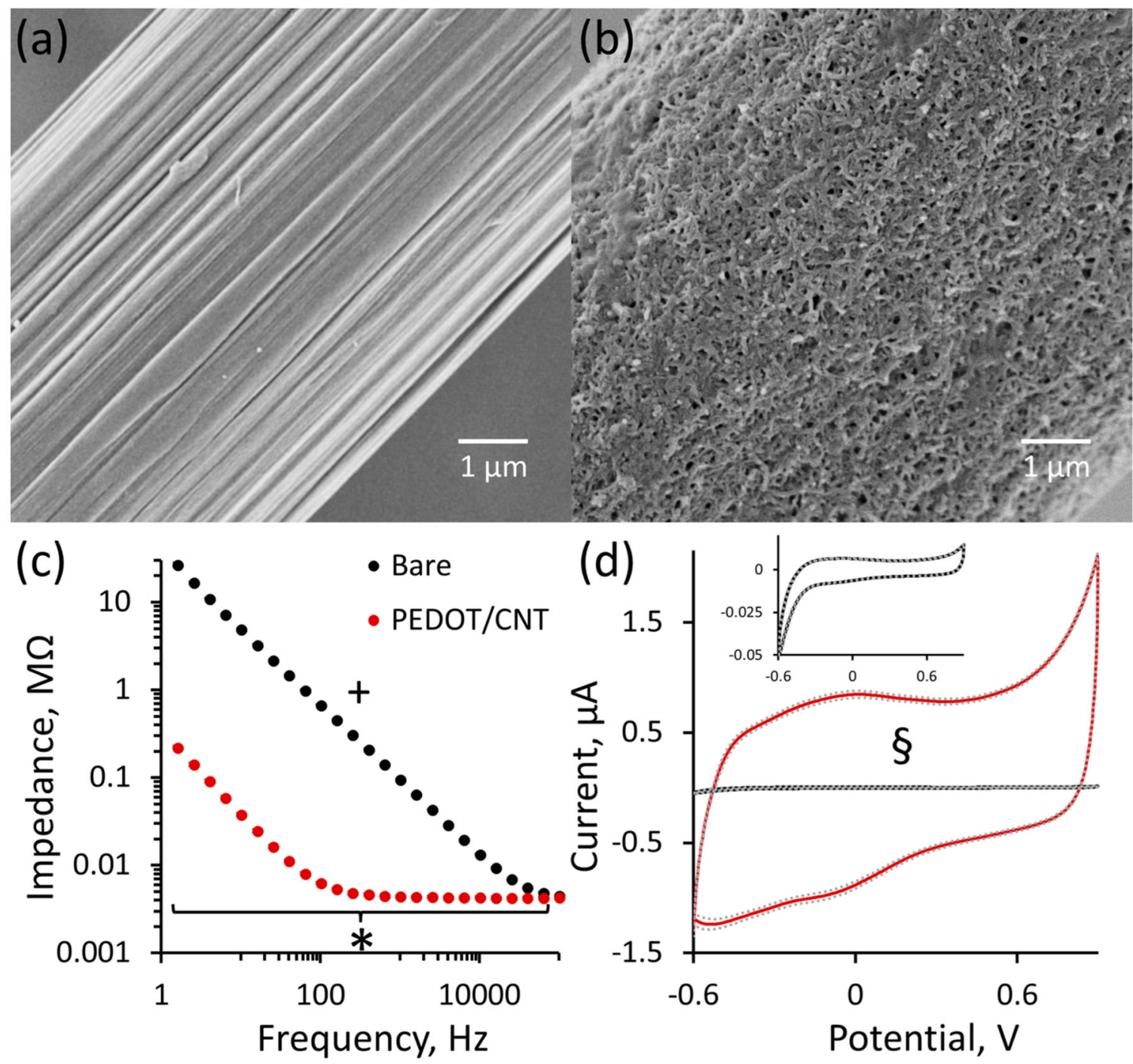

Figure 1.

Uniform PEDOT/CNT deposition onto CFEs significantly decreases impedance. Scanning electron microscopy images of representative bare (a) and $100 \mathrm{mC} / \mathrm{cm}^{2}$ PEDOT/CNTfunctionalized (b) CFEs. Comparison of electrode diameter for bare $(6.7 \mu \mathrm{m})$ and PEDOT/ CNT-functionalized $(9.6 \mu \mathrm{m})$ electrodes reveals the PEDOT/CNT coating to be approximately $1.5 \mu \mathrm{m}$ thick. (c) Average electrode impedance ( \pm SEM, $n=5)$ significantly changes with PEDOT/CNT coating and frequency, and those two variables exhibit a significant interaction (+ two-way ANOVA with repeated measures design: coating, $F(1.19,9.54)=1032, p<1 \times 10^{-9}$; frequency, $F(1.19,9.54)=424, p<2 \times 10^{-9}$; interaction, $\left.F(1.19,9.54)=410, p<5 \times 10^{-9}\right)$. Furthermore, PEDOT/CNT significantly decreases average impedance at each individual frequency below $100 \mathrm{kHz}$ (* Bonferroni post hoc analysis, $p<0.05$ ). (d) PEDOT/CNT coating significantly increases the average amplitude of the nonfaradaic charging current in response to a $1 \mathrm{~V} / \mathrm{s} \mathrm{CV}$ sweep ( \pm SEM dashed lines, $n$ $=5$ ) from $0.067 \pm 0.003$ to $3.40 \pm 0.09 \mu \mathrm{A}$. The inset displays the average bare response $\left( \pm\right.$ SEM, $\S$ one-way ANOVA, $\left.F(1,9)=1852, p<1 \times 10^{-10}\right)$. 


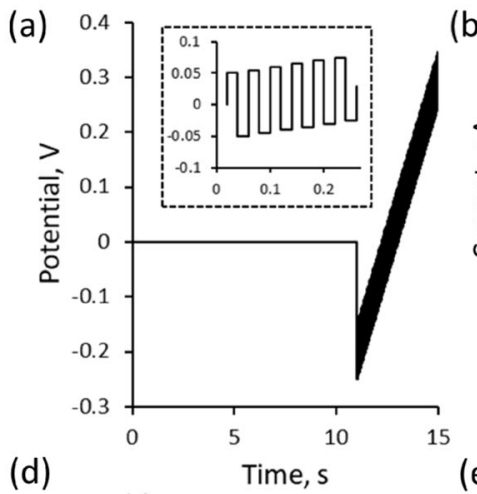

(b)
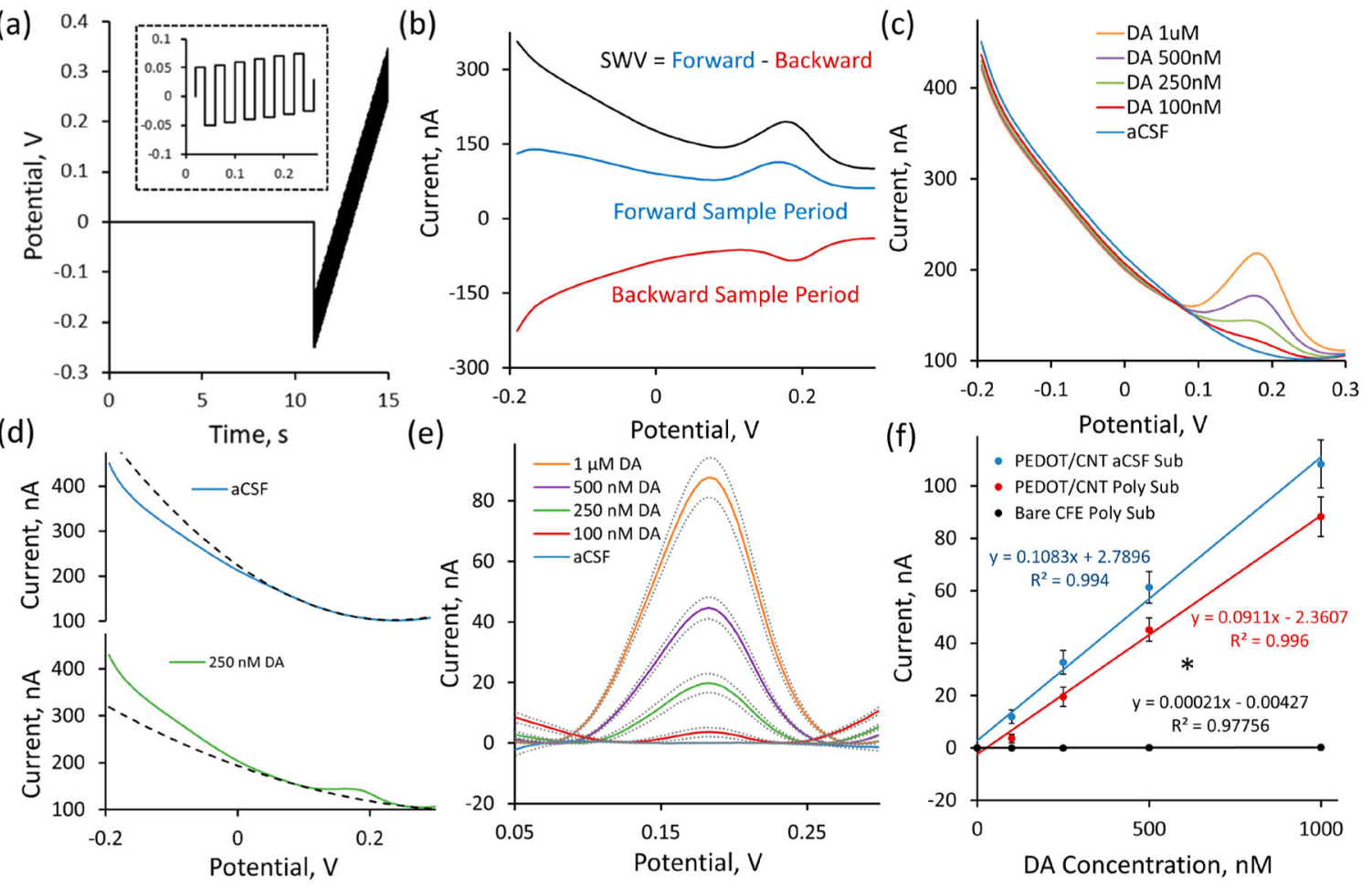

Figure 2.

PEDOT/CNT-functionalized CFEs are sensitive for resting DA via SWV. (a) The SWV waveform consists of an initial $0 \mathrm{~V}$ hold followed by a series of anodic and cathodic step and holds (zoom shown in the inset) that transverse the defined potential window. Forward current is measured at the end of each anodic hold period, and backward current is measured at the end of each cathodic hold period. (b) Representative SWV measurement of a $1 \mu \mathrm{M}$ standard solution of DA reveals the SWV current response to be the difference of the forward and reverse current responses. (c) Average ( $n=5$, SEM omitted for clarity) in vitro SWV DA calibrations conducted at PEDOT/CNT-functionalized CFEs in aCSF reveal clear DA peaks at $0.18 \mathrm{~V}$. (d) A second-order polynomial was used to model the background current for each SWV response within the potential region of interest (surrounding $E_{\text {peak }}$ $0.18 \mathrm{~V}$ ). The polynomial fit (dashed) produces a high-correlation fit within the potential region of interest to individual SWV traces (average SWV responses shown) both in the presence (250 nM DA, green) and absence (blue, aCSF) of DA. (e) Subtraction of the polynomial baseline fit reveals clear concentration-dependent Gaussian peaks (average \pm SEM) for solutions containing DA and nearly flat baselines in solutions where DA is absent. (f) Average DA sensitivity (background-subtracted peak current vs DA concentration) is linearly correlated. Incorporating PEDOT/CNT coating significantly increases DA sensitivity (* one-way ANOVA, $p<0.05$ ) from bare CFEs (black). Average sensitivity of PEDOT/CNT-functionalized CFEs following polynomial background subtraction (red) is not significantly different from the sensitivity obtained via aCSF subtraction (blue) (one-way ANOVA, $p>0.05$ ). 

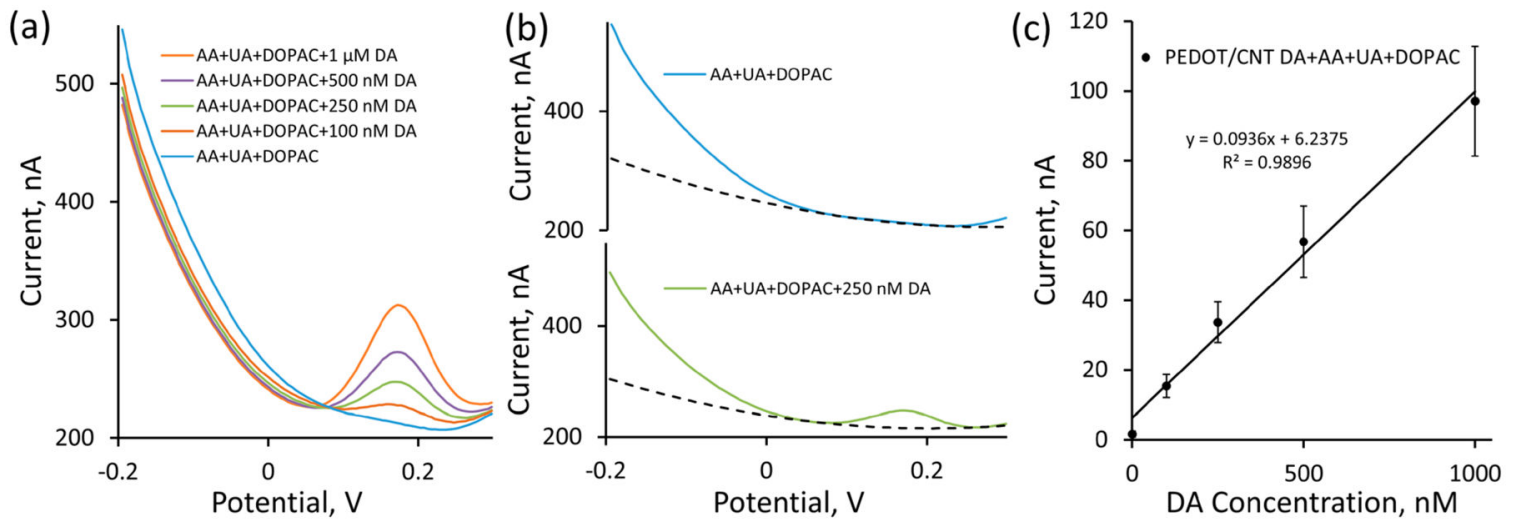

Figure 3.

PEDOT/CNT-functionalized CFEs are selective for resting DA via SWV. (a) Average ( $n=5$, SEM omitted for clarity) in vitro SWV DA calibrations conducted at PEDOT/CNTfunctionalized CFEs in the presence of a cocktail of common neurochemical interferences consisting of $200 \mu \mathrm{M} \mathrm{AA}, 10 \mu \mathrm{M} \mathrm{UA}$, and $10 \mu \mathrm{M}$ DOPAC (pH adjusted to 7.4) reveal clear DA peaks at $0.18 \mathrm{~V}$. (b) The second-order polynomial fit (dashed) was capable of modeling the baseline SWV response resulting from the interferences within the potential region of interest both in the presence (250 nM DA, green) and absence (blue) of DA. (c) Average DA sensitivity in the presence of interferences is linearly correlated. Incorporation of interfering agents does not produce a significant difference (one-way ANOVA, $p>0.05$, compared to Figure 2f, blue) in average DA sensitivity using SWV. 

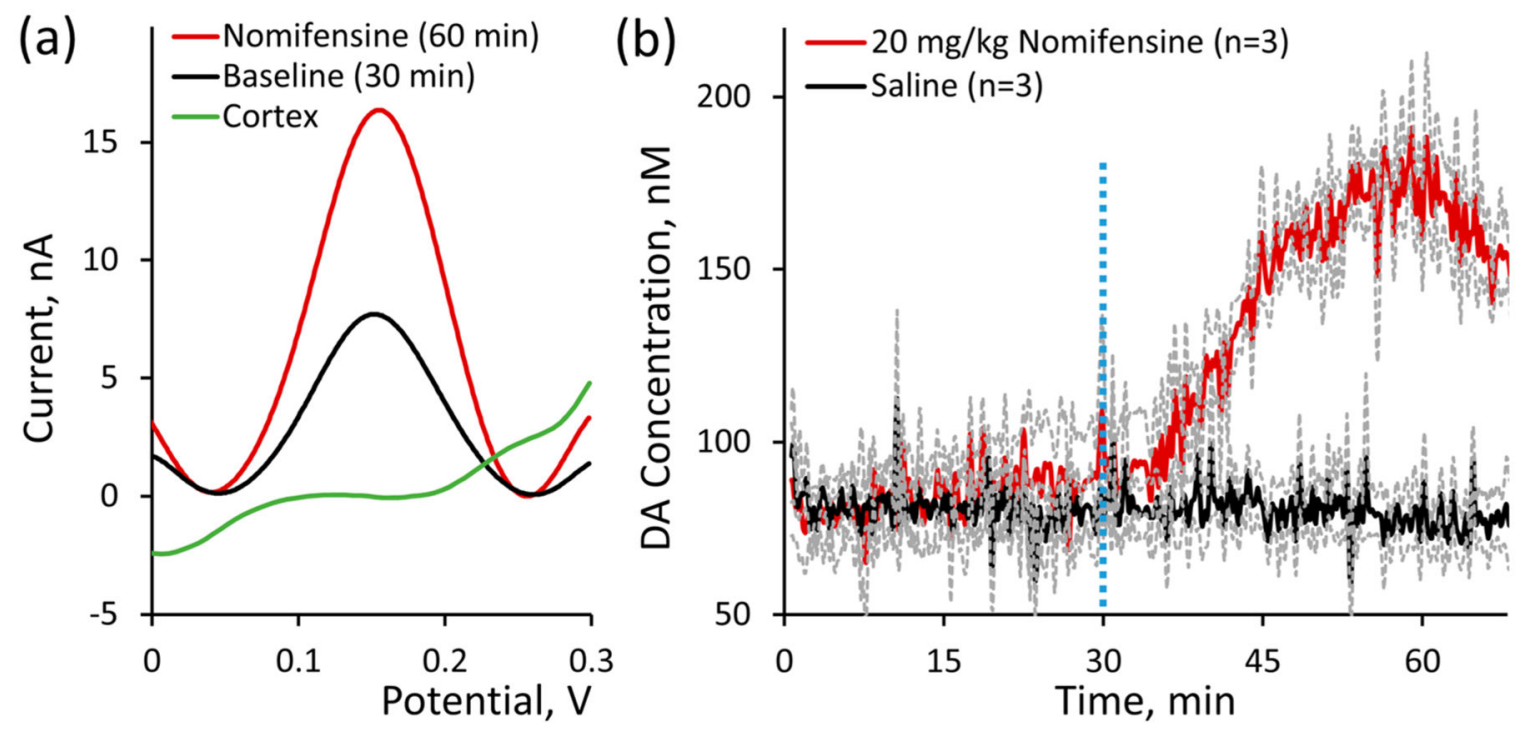

Figure 4.

PEDOT/CNT-functionalized CFEs are capable of directly measuring basal DA in vivo. (a) SWV measurements at PEDOT/CNT-functionalized CFEs reveal clear Gaussian DA peaks in the rat dorsal striatum ( $n=3$ average) but not in the non-DA-rich forelimb region of the primary somatosensory cortex (S1FL, $n=3$ average). (b) Average in vivo DA concentration $( \pm$ SEM) was plotted over time before and after ip administration of $20 \mathrm{mg} / \mathrm{kg}$ nomifensine (red, $n=3$ individual rats) or saline vehicle control (black, $n=3$ individual rats) at $t=30$ min (vertical dashed blue line). Basal DA was quantified on average to be $82 \pm 6 \mathrm{nM}$ ( $n=6$ individual rats), and nomifensine administration increased DA concentration beginning approximately 5 min following ip injection to a maximum DA concentration of $207 \pm 16 \mathrm{nM}$ at $28 \pm 2$ min following administration. 

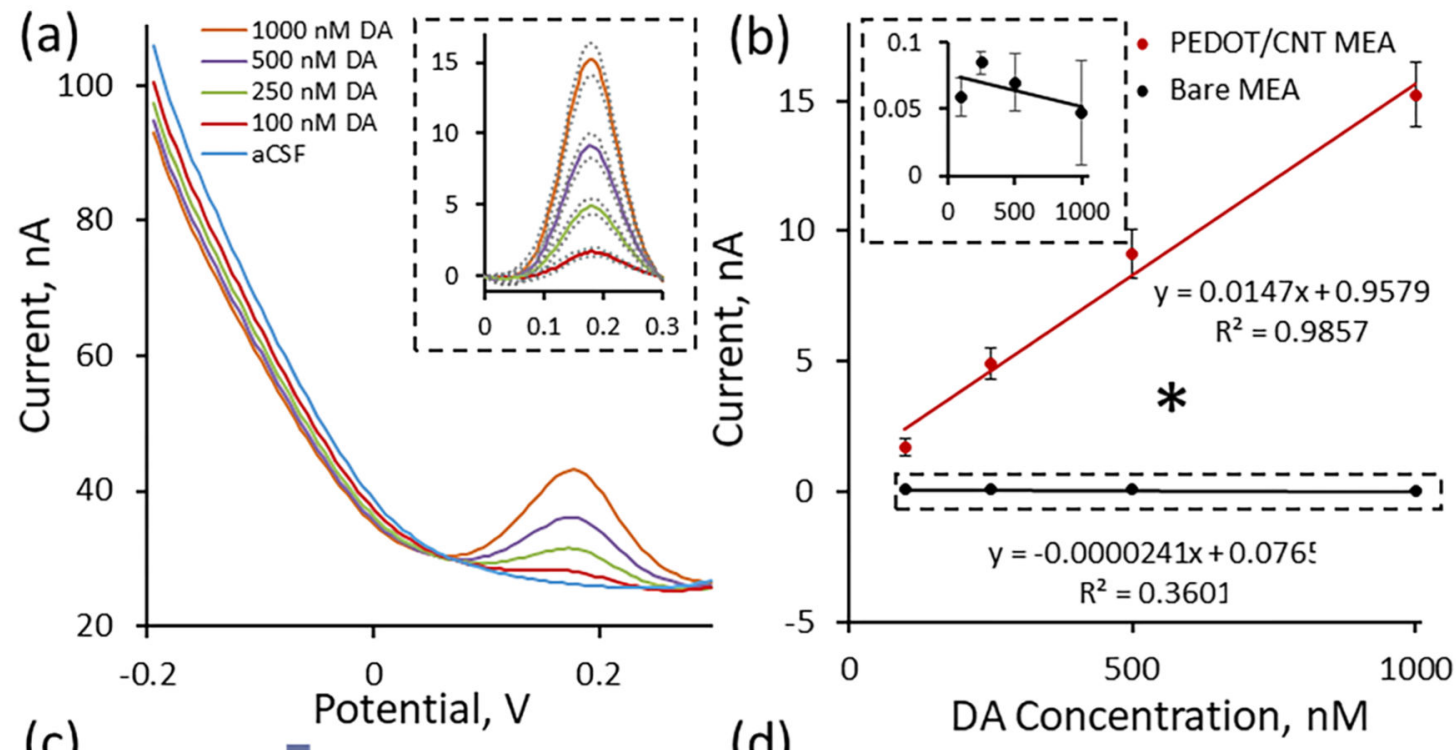

(c)

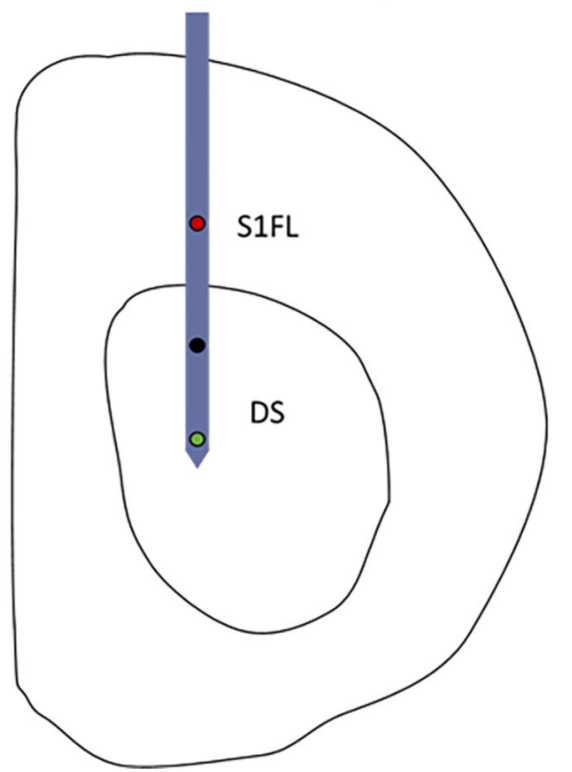

(d)

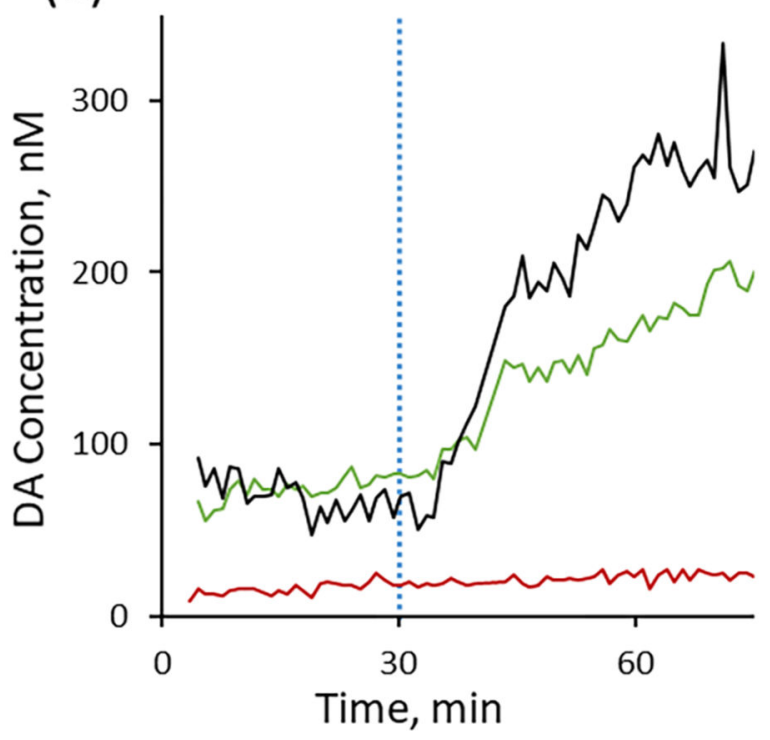

Figure 5.

PEDOT/CNT-functionalized MEAs are sensitive for resting DA. (a) Average ( $n=5$, SEM omitted for clarity) in vitro SWV DA calibrations conducted at PEDOT/CNT-functionalized MEAs in aCSF reveal a concentration-dependent DA peak at $0.18 \mathrm{~V}$. aCSF subtraction reveals the average ( \pm SEM) peaks to be Gaussian in shape (inset). (b) PEDOT/CNTfunctionalized MEAs exhibit linear sensitivity for DA $( \pm$ SEM). Uncoated gold MEA sites $( \pm \mathrm{SEM}, n=5$, isolated view shown in inset) are completely insensitive for DA detection, exhibiting both a nonlinear and negative sensitivity trend. PEDOT/CNT nanocomposite coatings significantly increase MEA sensitivity for DA (* one-way ANOVA, $p<0.05$ ). (c) Schematic drawing of in vivo MEA positioning. Two PEDOT/CNT-functionalized MEA electrode sites were located within the dorsal striatum (DS) (green and black), and one PEDOT/CNT-functionalized MEA electrode site was located within the non-DA rich S1FL (red). (d) Electrodes located within the DS (green and black) showed clear, nomifensine- 
dependent DA detection $(20 \mathrm{mg} / \mathrm{kg}$ ip injection at $t=30 \mathrm{~min}$, dashed blue vertical line $)$, whereas the electrode located within the S1FL (red) shows no nomifensine-dependent DA detection. 